\section{Odontodisplasia regional: a propósito de un caso}

\section{Regional odontodysplasia: a case report}

\section{Resumen}

La odontodisplasia regional (OR) es una anomalía del desarrollo poco frecuente, que involucra componentes del ectodermo y el mesodermo, esta alteración afecta tanto dentición temporal como permanente, siendo el maxilar frecuentemente más afectado que la mandíbula. Acude paciente masculino de 13 años por presentar retraso en la erupción de órganos dentarios 21 al 24. A la exploración intraoral los tejidos blandos contaban con características normales, se apreciaba una formación incompleta de los órganos dentarios sin lograr apreciar una distinción en la opacidad entre el esmalte y la dentina, con cámaras pulpares amplias. Se procedió a la extracción quirúrgica de los dientes 21 al 24, un mes posterior a esto se realizó la rehabilitación protésica temporal. La OR es una alteración poco descrita por lo que aún falta esclarecer la etiología y evolución a largo plazo del padecimiento, el dar a conocer los nuevos casos permite ampliar el conocimiento en cuanto a su frecuencia, además de concientizar al profesional de la salud acerca de la presencia y tratamiento de la misma.

Palabras clave: Ectodermo; Odontodisplasia; Odontología pediátrica.

\begin{abstract}
The regional odontodysplasia (RO) is a rare development anomaly that involves components of the ectoderm and mesoderm, this alteration affects both temporary and permanent dentition, being the maxilla more frequently affected than the mandible. A 13-year-old male patient presented delayed eruption of dental organs 21 to 24 . Intraoral examination of the soft tissues had normal characteristics, an incomplete formation of the dental organs was distinguished without achieving a distinction in the opacity between enamel and dentin, with wide pulp chambers. We proceeded to the surgical extraction of teeth 21 to 24 , a month after this, temporary prosthetic rehabilitation was performed. The RO is a little described alteration so it is still necessary to clarify the etiology and long-term evolution of the condition, the disclosure of new cases will allow to expand the knowledge regarding its frequency, as well as to raise awareness among the health professional about the presence and treatment of it.
\end{abstract}

Keywords: Ectoderm; Odontodysplasia; Pediatric dentistry.

\section{Caso Clínico}

Claudia Alejandra Ruezga-García ${ }^{1, a}$, Irma Mireya Balderrama-Armendáriz ${ }^{1, b}$, Cristina de la Peña-Lobato ${ }^{1, b}$,

Juan Carlos Cuevas-González 1,c

1 Universidad Autónoma de Ciudad Juárez. Instituto de Ciencias Biomédicas. Departamento de Estomatología. Juárez Chihuahua, México.

a Alumna.

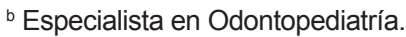

c Especialista en Patología y Medicina Bucal.

Correspondencia:

Juan Carlos Cuevas González

Correo electrónico:

cuevas_gonzalez@hotmail.com

Departamento de Estomatología, Universidad Autónoma de Ciudad Juárez. Anillo Envolvente del Pronaf s/n, Zona Pronaf, 32315 Cd Juárez Chihuahua, México.

\section{Coautores:}

Claudia Alejandra Ruezga-García ruezga_c@hotmail.com Irma Mireya Balderrama-Armendáriz irma.balderrama@uacj.mx Cristina de la Peña-Lobato cristina.delapena@uacj.mx

Conflicto de intereses: Los autores declaran no tener conflictos de interés.

Fuente de financiamiento: Autofinanciado.

Fecha de recepción: 28/10/18

Fecha de aceptación: 23/01/19

(c) Los autores. Este artículo es publicado por la revista Odontología Sanmarquina de la Facultad de Odontología, Universidad Nacional Mayor de San Marcos. Este es un artículo de acceso abierto, distribuido bajo los términos de la licencia Creative Commons Atribucion - No Comercia_Compartir Igual 4.0 Internacional. (http://creativecommons.org/licenses/by-nc-sa/4.0/) que permite el uso no comercial, distribución y reproducción en cualquier medio, siempre que la obra original sea debidamente citada. 


\section{Introducción}

La odontodisplasia regional (OR) fue descrita en 1934 por Hitchin, fue hasta 1963 que el término odontodisplasia fue propuesto por primera vez; se trata de una anomalía del desarrollo poco frecuente, que involucra componentes del ectodermo y el mesodermo ${ }^{1}$. Esta alteración afecta tanto dentición temporal como permanente, siendo el maxilar frecuentemente más afectado que la mandíbula sobre todo a la altura de los incisivos centrales y laterales ${ }^{2}$.

Se ha llegado a reportar una tasa menor a 1/1000 000 habitantes y solamente alrededor de 140 casos han sido publicados hasta el momento en la literatura ${ }^{3}$. Si bien la etiología no es del todo clara, se han considerado algunas hipótesis como son: trauma, deficiencias nutricionales, infecciones, anormalidades metabólicas, enfermedades sistémicas y defectos genéticos, aún no se ha establecido si realmente se encuentran implicados estos factores ${ }^{4}$, por lo que esta anomalía se encuentra aún en estudio.

Dentro de los diagnósticos diferenciales de la OR se encuentran la displasia dentinaria, dentinogénesis y amelogenesis imperfecta, algunas de las características clínicas de la OR pueden ser similares, sin embargo, estos desórdenes afectan la dentición de forma generalizada, cabe mencionar que una de las grandes diferencias es que el retraso de la erupción del órgano dentario solo se presenta en la odontodisplasia regional ${ }^{5,6}$. Al tratarse de un padecimiento poco frecuente, decidimos realizar este reporte de caso con el objetivo de identificar las principales características clínicas y radiográficas de la OR, para que de esta manera el odontólogo pueda identificar a este grupo de pacientes y conozca el manejo terapéutico de los mismos.

\section{Reporte del caso}

Paciente masculino de 13 años de edad que fue remitido al Posgrado de Odontopediatría de la Universidad Autónoma de Ciudad Juárez, Chihuahua, México, debido al retraso en la erupción de órganos dentarios centrales superiores $21,22,23$, y 24 . A la exploración física se observaba paciente con altura y peso adecuados para su edad, sin datos de asimetría facial, con implantación de cabello y piel normal, así como buena salud en términos generales; en la anamnesis los padres no mencionaron ningún antecedente de condición médica hereditaria o de algún desorden dental o genético en la familia.

A la exploración intraoral los tejidos blandos contaban con características normales, encía color rosado pálido, color de dientes erupcionados blanco aperlado, no refería dolor, y se podía notar el borde incisal del órgano dentario 21 a la altura del vestíbulo; en los estudios imagenológicos se advertía la vía aérea despejada, contorno óseo simétrico y sin alteraciones, senos maxilares neumatizados y cornetes inferiores hipertróficos; en el cuadrante superior izquierdo se distinguía una formación incompleta de los órganos dentarios sin lograr apreciar alguna variación en la opacidad entre el esmalte y la dentina con cámaras pulpares amplias; el paciente era portador de prótesis parcial removible (Figura 1). Se procedió a la extracción quirúrgica de los órganos dentarios 21 , 22, 23, 24 la cual se llevó a cabo sin complicaciones, un mes posterior a esto se realizó la rehabilitación protésica temporal (Figura 2 y 3 ).

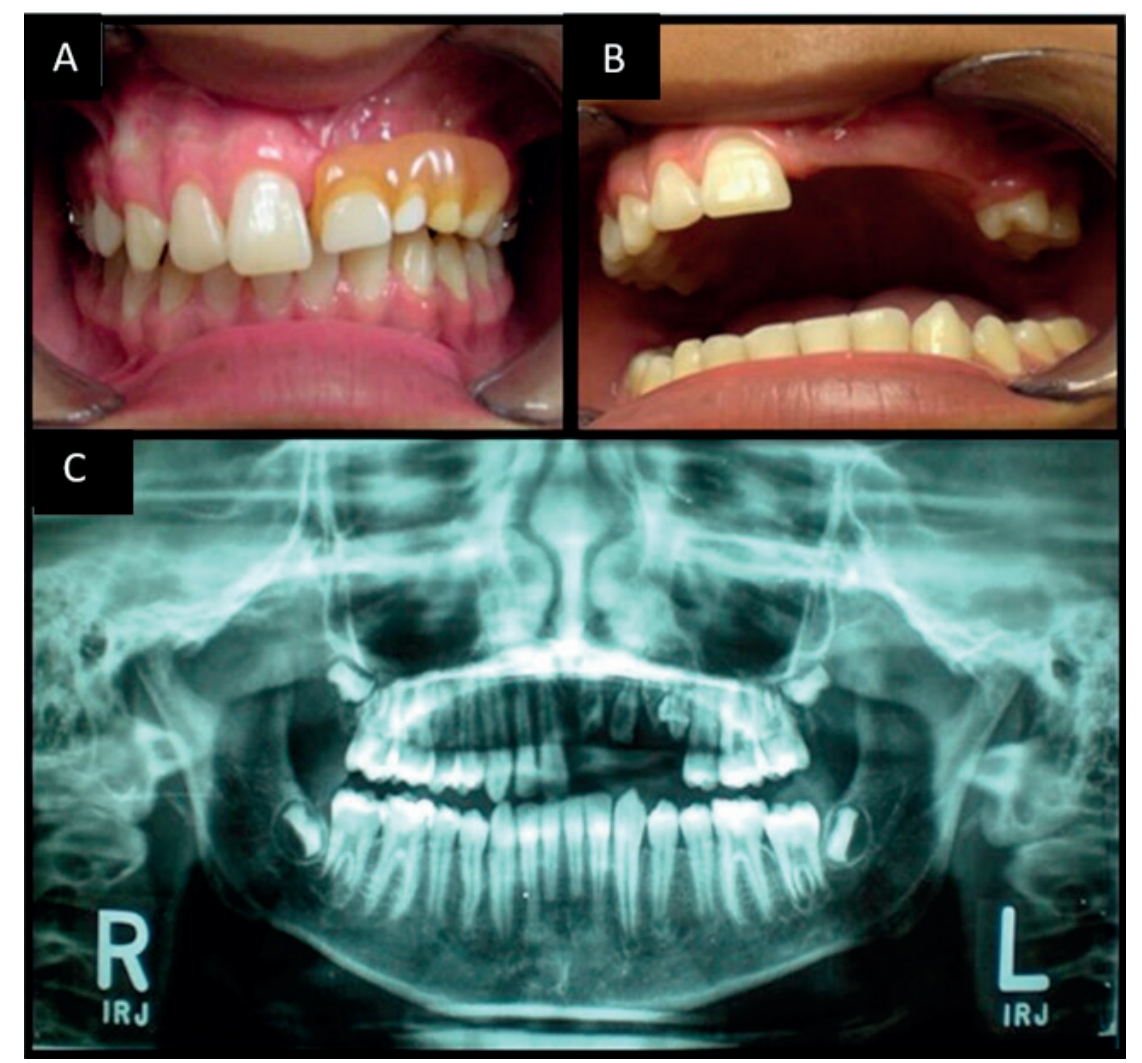

Figura 1. A. Prótesis parcial removible de más de 3 años de uso. B. Zona edéntula afectada por la odontodisplasia regional. C. Radiografía panorámica inicial para determinar tratamiento 


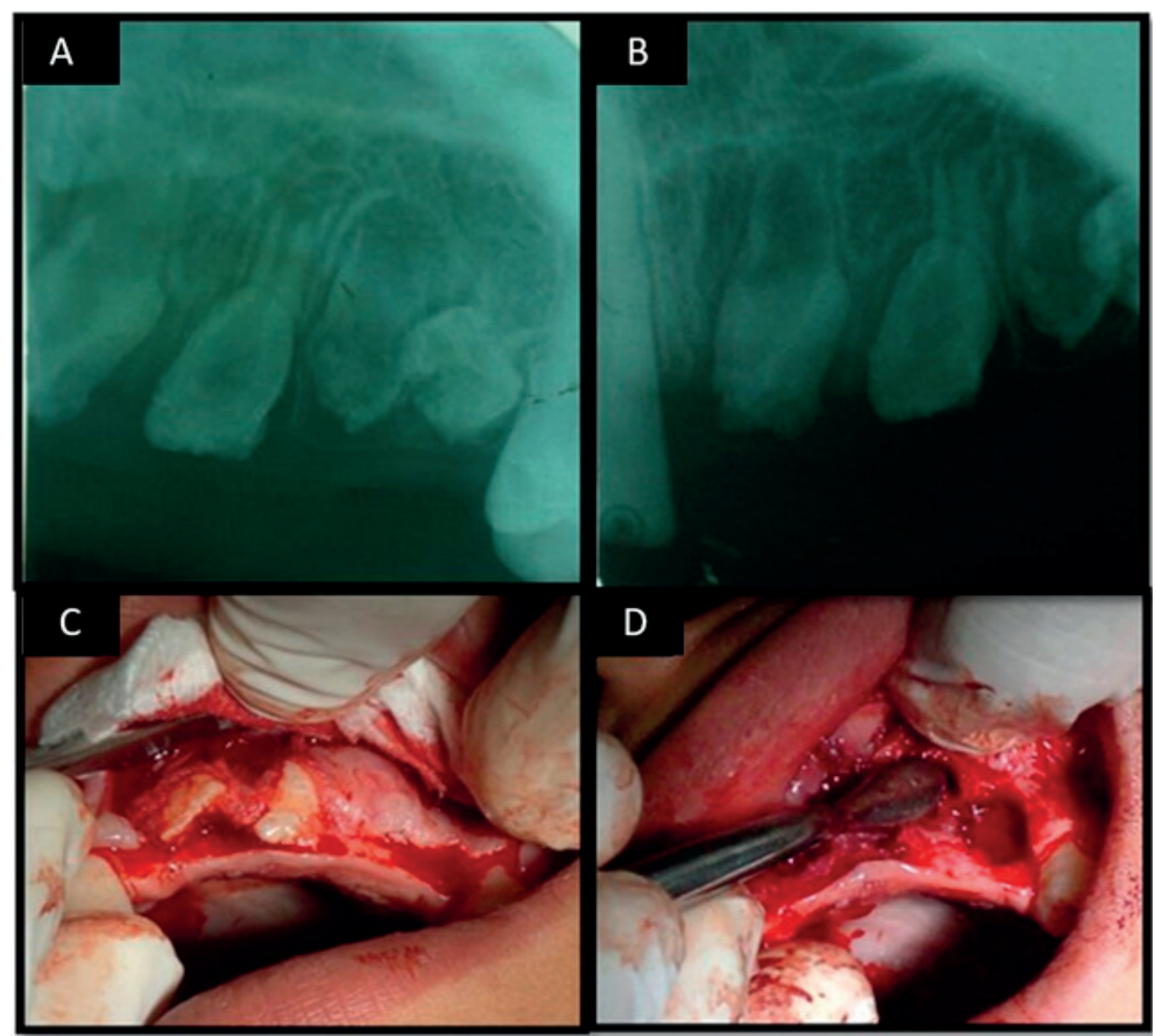

Figura 2. A y B. Radiografías periapicales donde se observa esmalte y dentina afectada. C. Extracción quirúrgica de dientes con odontodisplasia. D. Retiro de espículas en el área de la extracción

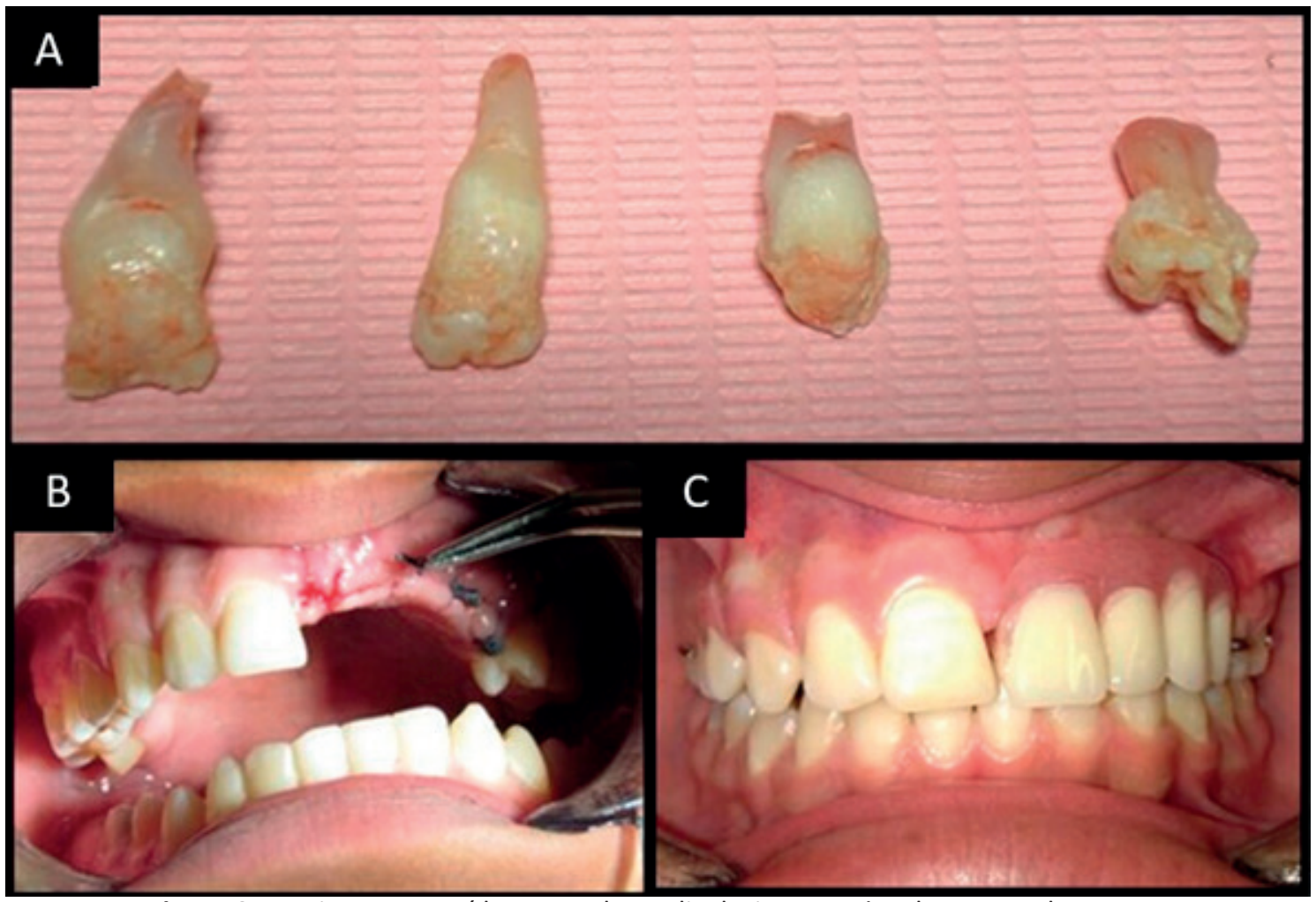

Figura 3. A. Dientes extraídos con odontodisplasia. B. Retiro de puntos de sutura.

C. Rehabilitación con prótesis parcial removible 


\section{Discusión}

La OR es una alteración de la cual se tiene poca información en la literatura, de acuerdo a lo reportado la edad de presentación es variable, sin embargo, esta patología se manifiesta durante la erupción de los dientes primarios o bien en dentición permanente, siendo más frecuente en pacientes del sexo femenino ${ }^{7}$, en nuestro caso la edad de presentación fue de 13 años, período que pertenece a la fase final del proceso de cambio de dentición temporal a permanente, por lo que coincidimos en lo reportado por la literatura. Referente al sexo, Mehta et al. ${ }^{1}$ mencionaron que las mujeres son más afectadas que los hombres, Babu et al. ${ }^{6}$ en el 2015 reportaron una tasa también mayor en el sexo femenino, contrario a nuestro caso que corresponde a un paciente de sexo masculino.

Los criterios diagnósticos de la OR están basados principalmente en las características clínicas y radiográficas ${ }^{1}$, dentro de las manifestaciones clínicas se encuentra el daño de un solo cuadrante con dentición temporal, permanente o mixta, hipo-calcificación e hipoplasia del esmalte y dentina, órganos dentarios pequeños, amorfos, con marcas en su superficie, con retraso en la erupción o incluso sin erupcionar debido a la falta de formación de la raíz ${ }^{8}$, también se ha descrito la presencia de hiperplasia gingival y órganos dentarios incluidos dentro del sobrecrecimiento gingival ${ }^{6}$, nuestro paciente clínicamente no mostraba crecimiento gingival o datos de hipoplasia e hipercalcificación del esmalte, por lo que el diagnóstico clínico no fue tan evidente.

Radiográficamente en la OR los dientes afectados muestran una morfología anormal, tienen apariencia de "dientes fantasma"; la cámara pulpar se aprecia alargada, con raíces cortas y ápices abiertos, la corona tipo cáscara es otra característica imagenológica de esta patología ${ }^{9}$, estas mismas características fueron también reportadas por Ganguly et al. ${ }^{10}$ en el 2012; nosotros coincidimos con lo antes mencionado al notar las mismas características radiográficas, las cuales fueron un apoyo para el diagnóstico.

El tratamiento de esta alteración depende de diversos factores como la edad del paciente, estado de salud general así como la cantidad y calidad de los órganos dentarios remanentes, sin embargo, gran parte de la literatura concuerda en preferir un tratamiento conservador; Gündüz et al. ${ }^{11}$ en el 2008 reportaron un caso en el cual el tratamiento se basó en la extracción de los órganos dentarios afectados y su posterior rehabilitación con prótesis parcial removible de acrílico, de la misma manera Magalhães et al. ${ }^{12}$ en el 2007 mencionaron el procedimiento realizado en una paciente de 5 años, el cual consistió en la extracción y rehabilitación de la zona afectada. Por otra parte, Cho ${ }^{13}$ señaló que una opción terapéutica es mantener los órganos dentarios erupcionados libres de infección en cavidad bucal para favorecer el desarrollo óseo del paciente.

Otro tratamiento es el propuesto por Ziegler et al. ${ }^{14} \mathrm{el}$ cual consiste en llevar a cabo procedimiento ortodónti- co y el autotransplante de órganos dentarios teniendo como resultados el devolver la oclusión funcional.

En el caso que presentamos el tratamiento estuvo acorde con lo reportado en la literatura, ya que retiramos los órganos dentarios afectados con la finalidad de evitar futuras complicaciones.

Debido a la edad del paciente se tomó la decisión de realizar una prótesis parcial removible con acrílico para mantener la integridad del reborde alveolar y que en un futuro se pudiera llevar a cabo la rehabilitación fija. Thimma Reddy et al. ${ }^{15}$ en el 2010 destacan que este tipo de tratamiento debe de estar enfocado en mantener la masticación, la dimensión vertical y estética adecuada.

Otro tratamiento que ha sido reportado en la literatura es el manejo endodóntico de dientes con necrosis y lesión periapical, para de esta manera favorecer el conservar los órganos dentarios en cavidad bucal ${ }^{16}$, si bien no existe consenso en la literatura con respecto a la elección del tratamiento, consideramos que cada caso debe de ser valorado de manera individual para optar por la mejor opción terapéutica.

Se concluye que, si bien los casos de odontodisplasia regional son poco frecuentes, es importante conocer las principales características clínicas del padecimiento, esto con el fin de identificar los casos que pudieran llegar a presentarse y elegir así la mejor opción de tratamiento que permita mejorar la calidad de vida de los pacientes.

\section{Referencias bibliográficas}

1. Mehta DN, Bailoor D, Patel B. Regional odontodysplasia. J Indian Soc Pedod Prev Dent. 2011;29(4):323-6.

2. Upadhyay V, Chaturvedi TP, Pandey RK, Chaurasia A, Singh P. Regional Odontodysplasia Crossing Midline: A Rare Case Report. Int J Clin Pediatr Dent. 2011;4(2):159-61.

3. Jahanimoghadam F, Pishbin L, Rad M. Clinical, Radiographic, and Histologic Evaluation of Regional Odontodysplasia: a Case Report with 5-year Follow-up. J Dent (Shiraz). 2016;17(2):159-63.

4. Matsuyama J, Tanaka R, Iizawa F, Sano T, Kinoshita-Kawano S, Hayashi-Sakai S, et al. Clinical and radiographic findings and usefulness of computed tomographic assessment in two children with regional odontodysplasia. Case Rep Dent. 2014. doi: 10.1155/2014/764393.

5. Kappadi D, Ramasetty PA, Rai KK, Rahim AM. Regional odontodysplasia: An unusual case report. J Oral Maxillofac Pathol. 2009;13(2):62-6.

6. Babu NS, Smriti RJ, Pratima DB. Regional odontodysplasia: Report of an unusual case involving mandibular arch. Contemp Clin Dent. 2015;6(2):237-9.

7. Hamdan MA, Sawair FA, Rajab LD, Hamdan AM, Al-Omari IK. Regional odontodysplasia: a review of the literature and report of a case. Int J Paediatr Dent. 2004;14(5):363-70.

8. Mathew A, Dauravu LM, Reddy SN, Kumar KR, Venkataramana V. Ghost teeth: Regional odontodysplasia of 
maxillary first molar associated with eruption disorders in a 10-year-old girl. J Pharm Bioallied Sci. 2015;7(Suppl 2): S800-3. doi: 10.4103/0975-7406.163570.

9. Rashidian A, Afsharian Zadeh M, Azarshab M, Zarrabian T. Regional Odontodysplasia: Report of a case. J Dent (Shiraz). 2013;14(4):197-200.

10. Ganguly R, Ramesh A. Regional odontodysplasia: a case of progressive tooth development. J Indian Soc Pedod Prev Dent. 2012;30(2):176-8.

11. Gündüz K, Zengin Z, Celenk P, Ozden B, Kurt M, Gunhan O. Regional odontodysplasia of the deciduous and permanent teeth associated with eruption disorders: A case report. Med Oral Patol Oral Cir Bucal. 2008;13(9):563-6.
12. Magalhães AC, Pessan JP, Cunha RF, Delbem AC. Regional odontodysplasia: case report. J Appl Oral Sci. 2007;15(6):465-9.

13. Cho SY. Conservative management of regional odontodysplasia: case report. J Can Dent Assoc. 2006;72(8):735-8.

14. Ziegler S, Neukam FW. Regional odontodysplasia: orthodontic treatment and transplantation of premolars. Am J Orthod Dentofacial Orthop. 2012;142(5):710-9.

15. Thimma Reddy BV, Vinay Reddy KK, Sunil B, Pujita R, Kiran K, Kranthi KR. Regional odontodysplasia. J Indian Soc Pedod Prev Dent. 2010;28(4):315-8.

16. Calle MA. Odontodisplasia regional: Manejo endodóntico. Rev. CES Odont. 2010;23(2):67-72. 
